\title{
Strong correlations in dense matter
}

\author{
D. Blaschke ${ }^{1,2,3, a}$, T. Fischer ${ }^{1}$, and L. Turko ${ }^{1}$ \\ ${ }^{1}$ Institute for Theoretical Physics, University of Wrocław, 50-204 Wrocław, Poland \\ ${ }^{2}$ Joint Institute for Nuclear Research, 141980, Dubna, Russia \\ ${ }^{3}$ National Research Nuclear University (MEPhI), 115409 Moscow, Russia
}

Received 28 November / Accepted 28 November 2020

Published online 21 December 2020

\begin{abstract}
We walk the reader through the selected research topics in the field of strong correlations in dense matter that are collected in this special issue, from their theoretical basis to applications in explaining heavy-ion collision experiments and superdense matter in neutron stars and their mergers.
\end{abstract}

This special issue of the European Journal of Physics: Special Topics gathers 26 articles that emerged from the 40th Max Born Symposium "Three Days on Strong Correlations in Dense Matter" which was organized jointly by the Institute of Theoretical Physics (University of Wroclaw), the Helmholtz International Centre for FAIR, and the Wroclaw Branch of the Polish Academy of Science. This Symposium took place in Wroclaw in the period October 9-12, 2019, and had an interdisciplinary character, addressing questions related with the phase structure of hot and dense matter determined by strong correlations such as bound states and condensates. These phenomena are studied in the theory and in phenomenological applications to thermonuclear fusion and heavy-ion collision experiments as well as to (exo)planets, supernova explosions, neutron stars, and their mergers. The main attention was devoted to the formation and dissociation of strong correlations under extreme conditions of temperature, density, and strong fields, with special emphasis on deconfinement and chiral symmetry restoration. The talks presented during the Symposium were concerning

- Hot and dense QCD matter theory: phase diagram, non-equilibrium phenomena, and equation of state,

- Hadron and lepton pair production in heavy-ion collisions,

- QCD phases in supernova collapse, compact stars and binary mergers,

- Compact Ultra-Dense Objects (CUDOS),

and covered also other related topics. The timetable with links to the slides of all talks can be found on the website of the Symposium [1]. As guest editors of this EPJ ST issue, we strived for an adequate presentation of the broad spectrum of excellent contributions that coined the atmosphere and the high scientific level of the meeting. Regrettably, the talk on "Matter under planetary interior conditions" with

a e-mail: david.blaschke@uwr.edu.pl 
an explicit relation to the Nobel prize in Physics 2019 that was announced during the Symposium, as well as the topic of CUDOS are not represented in this issue. To compensate for that, we could collect here contributions by colleagues who were invited but could not attend the Symposium.

In this Editorial Preface we would like to give an overview on the contents of the special issue that is structured as follows:

\section{Prolegomena}

2. Theory of strong correlations in dense matter

(a) Phases of matter

(b) Equations of state

(c) Nonequilibrium

\section{Heavy-ion collisions}

\section{Neutron stars and their mergers}

The Prologue of the present issue consists of the epistemological article by Jörg Hüfner that asks the question "Why was it possible to understand the quantum world?" [2]. It elucidates on the example of Max Planck's discovery of the radiation law how progress is made in science by applying the research method of trial and error and by introducing a quite moderate enlargement of the "knowledge space" with far-reaching consequences. This example is paradigmatic for the research field covered by this issue. Already in 1985 Rolf Hagedorn, the pioneer of the statistical description of particle production in collisions has entitled his contribution about the then 25 year old history of this field "How we got to QCD matter from the hadron side by trial and error" [3]. Such an epistemological discussion appears again in the review article by Marek Gazdzicki [4] who describes the last 20 years of the investigation of the phase diagram of strongly interacting matter in heavy-ion collision experiments at the CERN SPS as an example of Karl Popper's model for the process of gaining scientific understanding. According to Popper an initial problem situation is followed by a tentative theory (trial) and then by error elimination which results in a new problem situation.

After this short excourse on epistemology we would now like to guide the reader through the research topics that are covered in this special issue.

\section{Theory of strong correlations in dense matter}

The ongoing process of developing a theory of hot and dense matter is the central element of the Physics of strong correlations in dense matter, a field where the well developed methods of perturbation theory do not apply and even the degrees of freedom (quasiparticles) are not always well defined. Nonperturbative methods need to be developed and to this end the theory has to be developed by trial and error, guided by experiments.

The review by Craig Roberts and Sebastian Schmidt presents a modern view on the fundamental problem of the emergence of hadronic mass [5], putting the celebrated Nobel-prize winning concept of the Higgs mechanism of mass generation in the standard model into the perspective of the mass budget of the Universe. There it contributes only a negligible amount, while the generation of hadron masses by dynamical chiral symmetry breaking in QCD is responsible at least for most of the mass of visible matter. Such questions of cosmic dimension are linked to the structure of the pion and its parton distribution functions. 
The following article [6] by Wojciech Broniowski and Enrique Ruiz Arriola dwells on the subject of the (double) parton distribution functions of the pion and demonstrates the usefulness of the Nambu-Jona-Lasinio model as a simple field theory of the pion that captures the aspect of dynamical chiral symmetry breaking.

The dynamical quark mass generation mechanism is also the subject of the following article by Felix Spengler, Davide Campagnari and Hugo Reinhardt [7] who elaborate this within the Hamiltonian approach in Coulomb gauge QCD in $2+1$ dimensions and compare the results with their previous work in $d=3$ spatial dimensions. Such an approach is advantageous because it allows far-reaching analytical insights to the nonperturbative dynamical features of low-energy QCD, confinement and spontaneous breaking of chiral symmetry.

Many aspects of the low-energy QCD dynamics, such as the running of quark masses and the wave function renormalization can be addressed within a DysonSchwinger model that uses an effective, nonlocal separable interaction [8]. Such an approach has been generalized in the work by Davor Horvatic, Dalibor Kekez and Dubravko Klabucar [9] beyond the limit of isospin symmetry. This work is a preparation for investigating the breaking of the $U_{A}(1)$ symmetry as a characteristic feature of nonperturbative QCD not only in the vacuum but also at finite temperatures where this symmetry is expected to be restored, resulting in possible observable signatures.

While the chiral symmetry generally is a global symmetry which upon its dynamical breaking generates a massless Goldstone boson, an inhomogeneous realization of chiral symmetry breaking is favored at finite chemical potentials in the QCD phase diagram at low temperatures. Michael Buballa, Stefano Carignano and Lennart Kurth have studied in their article [10] the dependence of this pattern on the amount of explicit symmetry breaking, viz. the pion mass.

The next two contributions discuss the property of confinement in effective approaches. Michał Szymański uses the Polyakov loop and its direct relationship to the entropy for heavy quarks for defining a critical temperature of deconfinement, including its response to a strong external magnetic field [11]. The contribution by Jakub Jankowski [12] illustrates the properties of the entanglement entropy within a Hamilton-Jacobi formulation of holographic models of strongly coupled field theories, in particular as a probe of confinement which can be studied also in lattice gauge theories. Later in this issue we come back to the holographic formulation of strongly coupled field theories, when it is used by Rico Zöllner and Burkhard Kämpfer [13] to tackle the effect of heavy vector meson melting observed in heavy-ion collisions at the Large Hadron Collider (LHC).

We have stressed earlier in this Editorial Preface the interdisciplinary character of the problem of strong correlations in dense matter. Basic concepts for the diagnostics of the state of matter in heavy-ion collisions like the Mott mechanism of bound state dissociation were developed first in solid state physics and nonideal plasmas before they have been carried over to strongly interacting systems of nuclear matter and quark matter. The review article by Werner Ebeling, Heidi Reinholz and Gerd Röpke elucidates the role of charge screening and Pauli blocking for the composition and thermodynamic properties of nonideal hydrogen, helium and lithium plasmas at high pressures [14]. The equation of state (EoS) of these plasmas is essential for inertial fusion as a possible solution of mankind's energy production problems in the future and for the structure of giant planets or exoplanets, the discovery of which was honoured by awarding one half of the Nobel prize in Physics 2019 to Michel Mayor and Didier Queloz [15].

The EoS of nuclear matter with light cluster bound states has been derived in a well-known work by Typel et al. [16]. It is a symbiosis of a quantum statistical approach with a relativistic density functional theory that describes the Mott dissociation of these clusters due to Pauli blocking before the nuclear saturation density is reached. Stefan Typel discusses in his contribution to this special issue [17] 
a description of quasi-deuteron bound states above the nuclear saturation density within a generalization of the relativistic density functional theory that he uses for the interpretation of the effect of short-range correlations between nucleons.

In the theory of dense atomic and molecular gases, instead of the microphysical Pauli blocking effect, the concept of an eigenvolume of the particles with a hard-sphere repulsion is very successfully applied and leads to the Van der Waals description of real gases. A generalization of this concept, taking into account surface and curvature tensions in the EoS of hard spheres and hard discs is presented in the work by Nazar Yakovenko, Kyrill Bugaev, Larissa Bravina and Eugene Zabrodin [18]. It can be applied to atomic as well as to nuclear and hadronic systems.

The investigation of dense matter in laboratory experiments with collisions of high-energy particles concerns nonequilibrium processes such as particle production and transport phenomena. A kinetic description of pair production in time-dependent strong fields (the dynamical Schwinger effect) is outlined in the article by Ivan Aleksandrov, Vadim Dmitriev, Denis Sevostyanov and Stanislav Smolyansky [19] for the example of vacuum $e^{+} e^{-}$production in electric fields of arbitrary polarization. Shear viscosity and electrical conductivity as transport coefficients are considered in a quasiparticle model by Valeriya Mykhaylova [20]. Helmut Satz discusses the concept of self-organized criticality as a new approach to multihadron production in high-energy collisions [21].

\section{Heavy-ion collisions}

The review by Marek Gazdzicki [4] summarizes important results and problems that occurred in the study of the phase diagram of strongly interacting matter at the CERN SPS in the 20 years after the announcement that CERN's Heavy Ion programme has found evidence for the creation of a new state of matter [22]. It refers to the "trial and error correction" scheme of scientific progress and introduces a new dimension in the search for threshold phenomena that characterize the onset of a new state of matter. Besides the threshold for "onset of deconfinement" in the collision energy, there are indications for a threshold in the system size that would signal the "onset of fireball" that are particularly impressive in the accumulated data on the behavior of the $K^{+} / \pi^{+}$ratio to which the NA61 experiment has recently contributed.

The physical mechanism behind the "horn" in the energy dependence of the $K^{+} / \pi^{+}$ratio is still one of the challenging puzzles in the field. In the article by David Blaschke, Alexandra Friesen, Yuri Kalinovsky and Andrey Radzhabov [23] a description of the horn effect has been given that is based on the generalized BethUhlenbeck approach to the abundances of kaons and pions in hot, dense quark matter which suddenly freeze out from a nonequilibrium state that is characterized by a (medium dependent) pion chemical potential, signalizing pion Bose enhancement.

The article by Christian Spieles and Marcus Bleicher [24] reports on the relation of hadron spectra and flow observables to the underlying EoS that are obtained by one-fluid hydrodynamic simulations for FAIR/NICA energy range of $1 \mathrm{AGeV} \leq$ $E_{\text {lab }} \leq 10 \mathrm{AGeV}$. They investigate also the dependence on the initial conditions and details of the particlization. A hydrokinetic model is used by Yuri Sinyukov, Musfer Adzhymambetov and Volodymyr Shapoval to study the role of geometric sizes of colliding nuclei for the femtoscopic radii of the particle sources at LHC and top RHC energies [25].

A very hot topic in modern heavy-collision experiments is the production of light nuclear clusters. Their abundances can be described within the thermal statistical model with the same chemical freeze-out temperature as for the other hadron species that almost coincides with the QCD hadronization transition temperature $T_{c} \approx 155 \mathrm{MeV}$. This prompts the puzzling question: Are light nuclei produced directly 
from the quark-gluon plasma at the hadronization despite their tiny binding energy of a few $\mathrm{MeV}$ which is negligible in comparison to $T_{c}$ ? The expression "snowballs in hell" has been coined for this situation [26]. Stanislaw Mrowczynski discusses this situation by contrasting the early light nuclei production in the thermal model with the late one in the coalescence model, suggesting a comparative study of ${ }^{4} \mathrm{Li}$ vs. ${ }^{4} \mathrm{He}$ production for disentangling both scenarios [27].

Concluding this part on dense matter in heavy-ion collisions the reader finds the article by Rico Zöllner and Burkhard Kämpfer [13] who consider the former "smoking gun" signal of quark-gluon plasma formation, namely the melting of heavy vector mesons $(\Phi, J / \psi, \Upsilon)$, but now in a formulation that uses a holographic QCD-dual based on the famous AdS/CFT correspondence.

\section{Compact stars and their mergers}

Since neutron stars can be viewed as "gigantic atomic nuclei" with $A \approx 10^{57}$ nucleons, the ultimate heavy-ion collision is a merger of two neutron stars. The first event of this kind that has been observed by its gravitational wave signal as well as in all wavelength bands of the electromagnetic spectrum was GW170817 [28]. Undoubtedly, in such a merger event the most massive neutron stars in the Universe are formed with the most extreme densities in their interior. Therefore, Andreas Bauswein and Sebastian Blacker consider in their contribution [29] the question whether the likely occurrence of a quark deconfinement transition would leave a characteristic trace in the gravitational wave signal from such an event. They indeed find that the peak frequency at a given radius (tidal deformability) of the postmerger star is higher than in a purely hadronic scenario. For the first time they demonstrate that such a signal is also obtained when the merging stars are already hybrid stars with a quark matter core. It remains to observe a postmerger gravitational wave signal, which in the case of GW170817 unfortunately was not seen.

In the remainder of this final part of the special issue, five contributions relate EoS models to multimessenger data from observations of neutron stars and their mergers in order to find out how the most probable EoS for dense matter should look like. In the works $[30,31]$ an extended $\sigma-\omega$ model of nuclear matter is considered and it is shown that also without the consideration of quark deconfinement the astrophysical observations are consistent with nuclear matter properties at saturation density. A caveat of these two studies is that hyperons have not been considered which likely occur before the neutron star maximum masses above $2 M_{\odot}$ are reached, unless the threshold mass for quark deconfinement is even lower. In the contribution by Konstantin Otto, Micaela Oertel and Bernd-Jochen Schäfer [32] a nonperturbative functional renormalization group approach to the EoS of quark matter with two and three flavors has been developed and short hybrid star branches close to the maximum mass have been obtained. Michal Marczenko investigates the speed of sound for the recently introduced hybrid quark-meson-nucleon model that addresses the aspect of chiral symmetry restoration by parity doubling in the hadronic phase before quark deconfinement occurs [33]. Finally, Mateusz Cierniak and David Blaschke investigate a curiosity in hybrid star models, the special point in the mass-radius diagram of compact stars through which hybrid star models have to pass which differ just by the bag constant that determines the onset of deconfinement [34]. Varying the EoS for the hadronic phase does not affect the special point. The authors conclude from their study that a value less than $11.9 \mathrm{~km}$ as a result of the presently ongoing NICER radius measurement on the massive pulsar PSR J0740+6620 would indicate that this pulsar is a hybrid neutron star with deconfined quark matter in the inner core. 


\section{Epilogue}

On the cover of this special issue you find the Penrose tribar [35], an impossible object that we have chosen for the poster of the Max Born Symposium long before it was announced that Sir Roger Penrose will be awarded one half of the Nobel prize in Physics 2020 "for the discovery that black hole formation is a robust prediction of the general theory of relativity".

Glimpses of black holes appeared throughout our collection of contributions to the topic of strong correlations in dense matter. On the one hand as the real black holes through the question whether as result of a neutron star merger necessarily a black hole must form? Or, whether the $2.6 M_{\odot}$ compact object in the binary merger GW190814 was the heaviest neutron star or the lightest black hole? Questions, to which we have no answers yet, but which are directly related to the equation of state of dense nuclear matter! On the other hand black holes appeared as a mathematical tool via the AdS/CFT correspondence which links the temperature in a strongly coupled quantum field theory to the Hawking temperature of a black hole in its dual gravity theory. Such holographic models do belong now to the arsenal of paradigms that drive the evolution of the understanding of strong correlations in dense matter through trials and error elimination.

We acknowledge the support of the Max-Born Symposium by the University of Wroclaw, the Wroclaw Branch of the Polish Academy of Sciences, the Helmholtz International Center for FAIR, the Frankfurt Institute for Advanced Studies and Springer Publishing for supporting the Springer lecturer.

\section{References}

1. https://events.ift.uni.wroc.pl/event/61, accessed November 16, 2020

2. J. Hüfner, Eur. Phys. J. Special Topics 229, 3311 (2020)

3. R. Hagedorn, How we got to QCD matter from the hadron side by trial and error, CERN Report No. CERN-TH.3918/84 (1984)

4. M. Gazdzicki, Eur. Phys. J. Special Topics 229, 3507 (2020)

5. C.D. Roberts, S.M. Schmidt, Eur. Phys. J. Special Topics 229, 3319 (2020)

6. W. Broniowski and E. Ruiz Arriola, Eur. Phys. J. Special Topics 229, 3341 (2020)

7. F. Spengler, D. Campagnari, H. Reinhardt, Eur. Phys. J. Special Topics 229, 3351 (2020)

8. D. Blaschke, G. Burau, Y.L. Kalinovsky, P. Maris, P.C. Tandy, Int. J. Mod. Phys. A 16, $2267(2001)$

9. D. Horvatić, D. Kekez, D. Klabučar, Eur. Phys. J. Special Topics 229, 3363 (2020)

10. M. Buballa, S. Carignano, L. Kurth, Eur. Phys. J. Special Topics 229, 3371 (2020)

11. M. Szymański, Eur. Phys. J. Special Topics 229, 3387 (2020)

12. J. Jankowski, Eur. Phys. J. Special Topics 229, 3395 (2020)

13. R. Zöllner, B. Kämpfer, Eur. Phys. J. Special Topics 229, 3585 (2020)

14. W. Ebeling, H. Reinholz, G. Röpke, Eur. Phys. J. Special Topics 229, 3403 (2020)

15. https://www.nobelprize.org/uploads/2019/10/press-physics2019.pdf, accessed November 26,2020

16. S. Typel, G. Röpke, T. Klähn, D. Blaschke, H.H. Wolter, Phys. Rev. C 81, 015803 (2010)

17. S. Typel, Eur. Phys. J. Special Topics 229, 3433 (2020)

18. N. Yakovenko, K. Bugaev, L. Bravina, E. Zabrodin, Eur. Phys. J. Special Topics 229, $3445(2020)$

19. I.A. Aleksandrov, V.V. Dmitriev, D.G. Sevostyanov, S.A. Smolyansky, Eur. Phys. J. Special Topics 229, 3469 (2020)

20. V. Mykhaylova, Eur. Phys. J. Special Topics 229, 3487 (2020) 
21. H. Satz, Eur. Phys. J. Special Topics 229, 3497 (2020)

22. U.W. Heinz, M. Jacob, arXiv:nucl-th/0002042 (2000)

23. D. Blaschke, A. Friesen, Y.L. Kalinovsky, A. Radzhabov, Eur. Phys. J. Special Topics 229, 3517 (2020)

24. C. Spieles, M. Bleicher, Eur. Phys. J. Special Topics 229, 3537 (2020)

25. Y. Sinyukov, M. Adzhymambetov, V. Shapoval, Eur. Phys. J. Special Topics 229, 3551 (2020)

26. D. Oliinychenko, L.G. Pang, H. Elfner, V. Koch, Phys. Rev. C 99, 044907 (2019)

27. S. Mrowczynski, Eur. Phys. J. Special Topics 229, 3559 (2020)

28. B.P. Abbott et al., Astrophys. J. Lett. 848, L12 (2017)

29. A. Bauswein, S. Blacker, Eur. Phys. J. Special Topics 229, 3595 (2020)

30. G.G. Barnaföldi, P. Pósfay, B.E. Szigeti, A. Jakovác, Eur. Phys. J. Special Topics 229, 3605 (2020)

31. D. Alvarez-Castillo, A. Ayriyan, G.G. Barnaföldi, H. Grigorian, P. Pósfay, Eur. Phys. J. Special Topics 229, 3615 (2020)

32. K. Otto, M. Oertel, B.-J. Schaefer, Eur. Phys. J. Special Topics 229, 3629 (2020)

33. M. Marczenko, Eur. Phys. J. Special Topics 229, 3651 (2020)

34. M. Cierniak, D. Blaschke, Eur. Phys. J. Special Topics 229, 3663 (2020)

35. L.S. Penrose, R. Penrose, Br. J. Psychol. 49, 31 (1958) 